\title{
CAMBIOS Y TENDENCIAS EN EL NÚMERO DE DÍAS DE LLUVIA EN LA REGIÓN DE MURCIA (1947-2016) Víctor Ruiz Álvarez ${ }^{(1)}$, Francisco Belmonte Serrato ${ }^{(2)}$, Ramón García Marín ${ }^{(3)}$ \\ (1) Departamento de Geografía, Universidad de Murcia. Campus de la Merced, 30001 victor.ruiz1@um.es (2) Departamento de Geografía, Universidad de Murcia, Campus de la Merced, 30001 franbel@um.es \\ (3) Departamento de Geografía, Universidad de Murcia, Campus de la Merced, 30001 ramongm@um.es
}

\section{RESUMEN}

En este trabajo se analizan los cambios y las tendencias en el número de días de lluvia en el ámbito territorial de la Región de Murcia (sureste de la península ibérica), durante el periodo temporal 1947-2016. Se han seleccionado los índices climáticos de precipitación, recomendados por la Organización Meteorológica Mundial (OMM) para el estudio de las tendencias del número de días de precipitación. Estos índices son: día de lluvia (precipitación $\geq 1 \mathrm{~mm}$ ); días de precitación $\geq 10 \mathrm{~mm}$ (DP10, número anual de días en que la precipitación es $\geq 10 \mathrm{~mm}$ ); días de precipitación abundante (DP20, número anual de días en que la precipitación es $\geq 20 \mathrm{~mm}$ ); días de precipitación muy abundante (DP50, número anual de días en que la precipitación es $\geq 50 \mathrm{~mm}$ ). Se han utilizado un total de 41 series de precipitación diaria distribuidas a lo largo del territorio de la Región de Murcia. Estas series han sido previamente reconstruidas y homogeneizadas bajo los criterios establecidos por la OMM, para el análisis de las series climáticas.

Para la evaluación de la significación estadística ( $\mathrm{p} \leq 0,05$, nivel de confianza del 95\%) de las tendencias se ha empleado el test no paramétrico de Mann-Kendall (Kendall,1976). Se han calculado tendencias anuales y estacionales. Se ha obtenido, una tendencia anual positiva en el número de días de lluvia ( $\geq 1 \mathrm{~mm}$ ), en la mayoría de los observatorios analizados, siendo significativamente positiva en algunas series del interior del territorio analizado. En cambio, en el resto de intervalos de precipitación diaria, la tendencia es claramente negativa, siendo este descenso estadísticamente significativo en la mayor parte de las series analizadas, en los intervalos de precipitación abundante y muy abundante (precipitación $\geq 20$ y $\geq 50 \mathrm{~mm}$ ). También se observa un cambio del máximo pluviométrico estacional desde la primavera al otoño. En definitiva, estos cambios en el número de días de lluvia podrían implicar numerosos impactos territoriales y socioeconómicos, por lo que resulta de vital importancia la puesta en marcha de medidas de mitigación y adaptación, más si cabe en ámbitos semiáridos.

\section{INTRODUCCIÓN}

En este estudio se analizan los cambios y las tendencias en el número de días de lluvia en el ámbito territorial de la Región de Murcia (sureste de la península ibérica), durante el periodo temporal 1947-2016. Estos cambios podrían implicar numerosos impactos territoriales y socioeconómicos, por lo que resulta de vital importancia la puesta en marcha de medidas de mitigación y adaptación, más si cabe en ámbitos semiáridos, como la Región de Murcia. En esta Región, y en general en todo el SE peninsular, estos estudios tienen una enorme trascendencia e importancia debido a la elevada variabilidad 
interanual que caracteriza a su clima, de modo que se suceden largos periodos secos, con episodios de lluvias breves pero muy intensas, que causan graves inundaciones (MartínVide y Olcina Cantos, 2001).

Dentro de una escala mensual, se han llevado a cabo numerosos estudios y trabajos sobre las tendencias de las precipitaciones durante el último siglo. En el ámbito mediterráneo, los análisis realizados reflejan una tendencia descendente de las precipitaciones durante la segunda mitad del S.XX, así como una intensificación de los periodos de sequía (Hoerling et al., 2012, Sousa et al., 2011). En Hoerling et al., (2011) se expone la existencia de un aumento de la intensidad y frecuencia de las sequías y rachas secas en las regiones mediterráneas a partir de la década de 1970. En el ámbito de la península ibérica, los estudios realizados sobre las tendencias y evolución de las precipitaciones también reflejan un descenso de las mismas desde la segunda mitad del S.XX para gran parte de su territorio, incluyendo la mitad oriental, y abarcando la mayor parte de estos trabajos más de 100 años de registros (Esteban Parra et al., 1998; Serrano et al., 1999; Brunet et al., 2007; Gallego et al., 2011; Ríos Cornejo et al., 2013). Este hecho se encuentra en consonancia con lo afirmado en el Quinto Informe de Evaluación del Grupo Intergubernamental de Expertos sobre el Cambio Climático (AR5).

A nivel regional, también se han estudiado las tendencias de las precipitaciones durante las últimas décadas, así como la posible intensificación de las sequías y el impacto de estas sobre el territorio (García Marín y Conesa García, 2006; García Marín y Calvo García-Tornel, 2008; Sánchez Toribio et al., 2010). En estos estudios de ámbito regional se habla de la acentuación e intensificación de los periodos secos en el SE peninsular desde mediados del S. XX. García Marín y Calvo García-Tornel (2008) muestran que las rachas secas se manifiestan como uno de los hechos climáticos definidores del ambiente mediterráneo, y su transcendencia es sustancial en el funcionamiento de muchos ecosistemas, al generar fuertes alteraciones en la disponibilidad de agua dentro de un ámbito semiárido. En Ruiz Álvarez et al. (2017) se muestra que la tendencia anual de las precipitaciones en la Región de Murcia apenas ha variado en los últimos 60 años, evidenciándose un aumento significativo de las precipitaciones en el mes de septiembre $\mathrm{y}$ un descenso significativo en el mes de junio.

A escala de precipitación diaria se han realizado también numerosos estudios dentro del ámbito de la península ibérica. De Luis et al. (2000) exponen que las áreas del interior de la Comunidad Valenciana presentan una disminución significativa de la precipitación anual, asociada con un descenso en el número de días de lluvia y un aumento de la variabilidad interanual de las precipitaciones. En cambio, en el litoral de la Comunidad Valenciana no se pueden definir cambios significativos. En Miró et al. (2009) se analizan las tendencias de precipitación diaria en las Demarcaciones Hidrográficas del Segura y Júcar, durante el periodo temporal 1958-2008. Los resultados obtenidos muestran tendencias claras al descenso de las precipitaciones de origen atlántico, y un aumento del porcentaje de las precipitaciones de origen mediterráneo. Por otra parte, González Hidalgo et al. (2003) analizan las tendencias de precipitación diaria en la Comunidad Valenciana durante el S. XX, y los resultados obtenidos confirman la hipótesis de que las variaciones anuales de precipitaciones dependen de los cambios que se localizan en 
unos pocos eventos lluviosos. Además, a pesar de su tendencia negativa, los eventos de precitación abundante y muy abundante ( $\geq 20 \mathrm{~mm}$ y $\geq 50 \mathrm{~mm}$ respectivamente) han aumentado su contribución a la precipitación anual.

Por último, en las proyecciones regionalizadas de cambio climático para el S.XXI realizadas por AEMET, en la Región de Murcia se estima un descenso entre 5-10 días en el número de días de lluvia. Igualmente se vislumbra un aumento entre 5-15 días en la duración de los periodos secos y un descenso entre un 5-15\% en la precipitación anual. En las precipitaciones intensas no hay una tendencia marcada (figura 1).
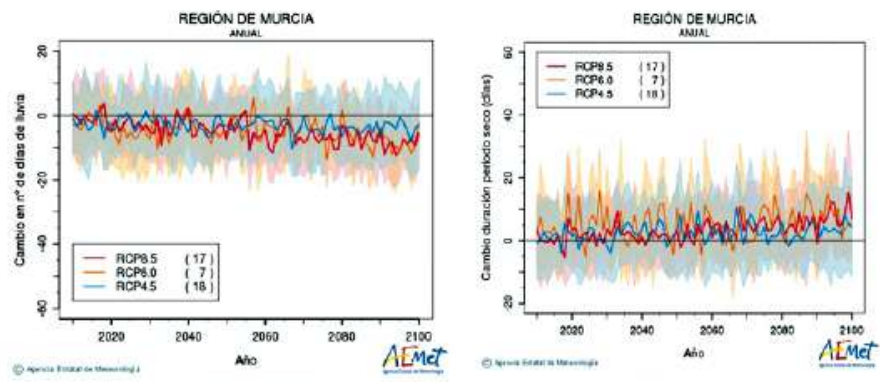

Fig. 1. Proyecciones regionalizadas de cambio climático para el siglo XXI. Cambios en la precipitación en la Región de Murcia. Fuente: Agencia Estatal
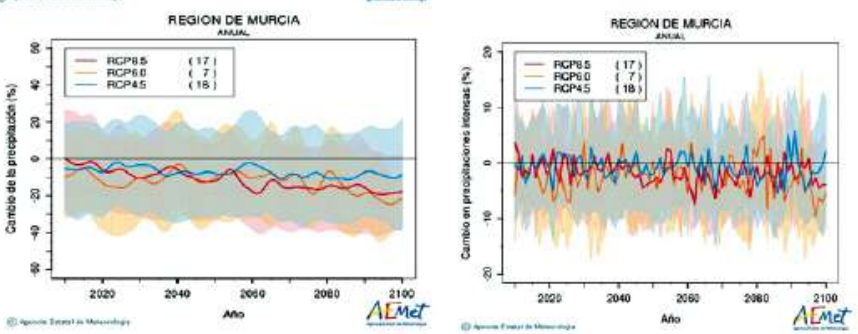
de Meteorología (AEMET).

\section{METODOLOGÍA}

El área de estudio de este trabajo es la Región de Murcia, situada en el sureste de la península ibérica (figura 2). Este territorio cuenta con una elevada variabilidad interanual en las precipitaciones. Además, la distribución espacial de las mismas presenta una gran heterogeneidad, pasando de una precipitación anual de más de $600 \mathrm{l} / \mathrm{m}^{2}$ en el extremo noroccidental, a menos de $200 \mathrm{~m} / \mathrm{l}^{2}$ en el extremo suroccidental. Este enclave es una de las áreas más áridas del continente europeo. El extremo noroccidental está influenciado por las precipitaciones de origen atlántico, y en este se localizan los embalses de mayor capacidad de la Demarcación Hidrográfica del Segura (DHS). Estos embalses se encuadran en el límite provincial entre las provincias de Albacete y Murcia.

La Región de Murcia tiene una superficie de $11.313 \mathrm{~km}^{2}$. En este estudio, se han seleccionado un total de 41 series de precipitación diaria procedentes de la base de datos de la Agencia Estatal de Meteorología (AEMET), que se encuentran distribuidas espacialmente a lo largo del territorio analizado (figura 2). Estas series han sido previamente 
reconstruidas y homogeneizadas bajo los criterios establecidos por la OMM para el análisis de las series climáticas (Mestre et al., 2013). El periodo temporal seleccionado es 1947-2016, es decir, se han analizado las tendencias diarias de las precipitaciones durante los últimos 70 años.

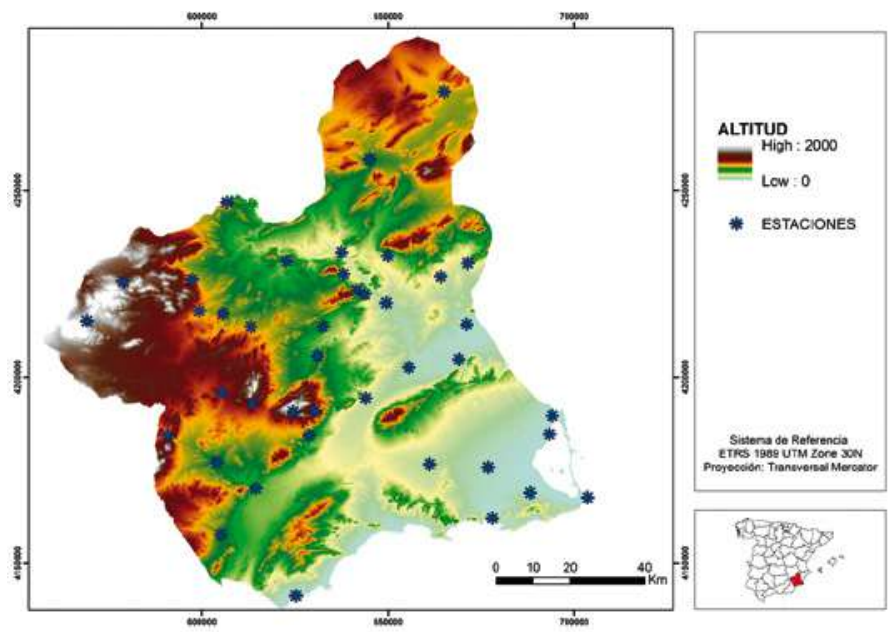

Fig. 2. Área de estudio y localización de las estaciones meteorológicas

A simis mo, se han seleccionado los índices climáticos de precipitación recomendados por la Organización Meteorológica Mundial (OMM) para el estudio de las tendencias del número de días de precipitación. Estos índices son: día de lluvia (precipitación $\geq 1 \mathrm{~mm}$ ); días de precitación $\geq 10 \mathrm{~mm}$ (DP10, número anual de días en que la precipitación es $\geq 10 \mathrm{~mm}$ ); días de precipitación abundante (DP20, número anual de días en que la precipitación es $\geq 20 \mathrm{~mm}$ ); días de precipitación muy abundante (DP50, número anual de días en que la precipitación es $\geq 50 \mathrm{~mm}$ ). Se han calculado las tendencias en estas variables para cada una de las series analizadas, tanto a escala anual como a escala estacional. Para la evaluación de la significación estadística ( $\mathrm{p} \leq 0,05$, nivel de confianza del 95\%) de las tendencias se ha empleado el test no paramétrico de Mann-Kendall (Kendall, 1976). Posteriormente, las tendencias han sido representadas espacialmente, para poder vislumbrar de un modo preciso y conciso la variabilidad existente en el territorio analizado.

\section{RESULTADOS Y DISCUSIÓN}

En primer lugar, se presentan los resultados obtenidos sobre las tendencias en el número de días de lluvia en las 41 series analizadas, tanto a escala anual como a escala estacional. Los resultados se muestran representados espacialmente, con el objetivo de reflejar la variabilidad territorial de las tendencias, y si estas presentan significancia estadística. Por último, se exponen como ejemplo los cambios observados en la serie "Moratalla Campo de San Juan", estación situada a 1150 metros de altitud y localizada en el extremo noroccidental de la Región de Murcia. Se ha seleccionado esta serie debido a que en estas zonas de interior se están produciendo los cambios de precipitación diaria más significativos. Como ya ha sido mencionado anteriormente, en esta zona se localizan la mayor parte de las reservas hídricas de la Demarcación Hidrográfica del Segura. 
Por lo tanto, el descenso en el número de días de lluvia y el aumento de los periodos secos, estimado por las proyecciones de las proyecciones de cambio climático para el S.XXI, puede incrementarlos ya numerosos conflictos sociopolíticos en este territorio, ya que de este enclave depende el abastecimiento de recursos hídricos, del resto del territorio de la Región de Murcia.

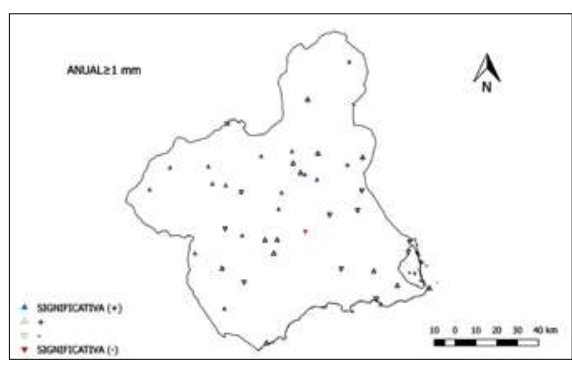

Fig. 4. Tendencia anual en el número de días de precipitación $(\geq 10 \mathrm{~mm}$ ) durante el periodo 1947-2016

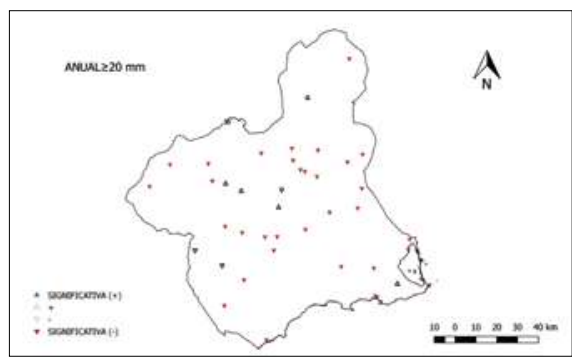

Fig. 6. Tendencia anual en el número de días de precipitación muy abundante $(\geq 50 \mathrm{~mm})$ durante el periodo 1947-2016
Fig. 3. Tendencia anual en el número de días de lluvia ( $\geq 1 \mathrm{~mm}$ ) durante el periodo 1947-2016

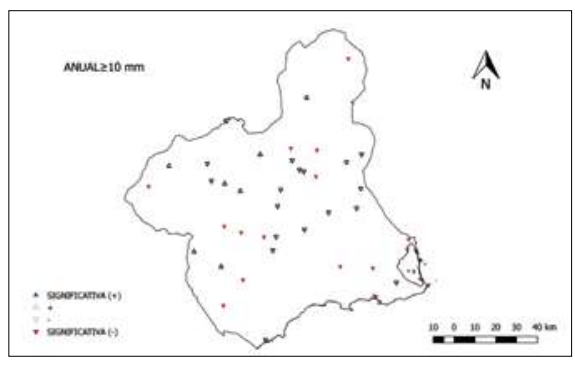

Fig. 5. Tendencia anual en el número de días de precipitación abundante $(\geq 20 \mathrm{~mm})$ durante el periodo 1947-2016

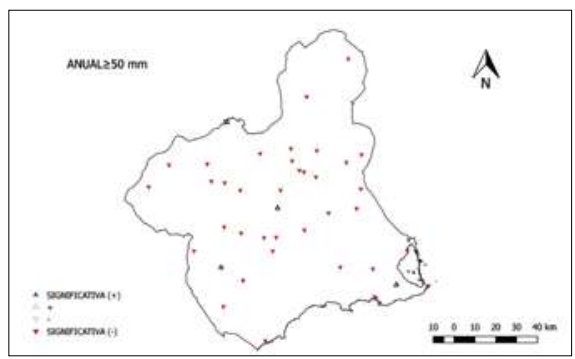

Desde la figura 3 a la figura 6, se muestran los resultados obtenidos de la tendencia anual de los días de precitación para los diferentes intervalos seleccionados. Se ha obtenido una tendencia positiva en el número de días de lluvia $(\geq 1 \mathrm{~mm})$ en la mayor parte de los observatorios analizados, siendo significativamente positiva en algunas series del interior de la Región de Murcia. En cambio, en el resto de intervalos de precipitación diaria la tendencia es claramente negativa, siendo este descenso estadísticamente significativo en la mayor parte de las series analizadas, y en los intervalos de precipitación abundante y muy abundante (precipitación $\geq 20 \mathrm{~mm}$ y $\geq 50 \mathrm{~mm}$ ). Este descenso significativo ha 
sido observado también en regiones vecinas como la Comunidad Valenciana (González Hidalgo et al., 2003; De Luis et al., 2000). El aumento de los días de precipitación $\geq 1 \mathrm{~mm}$ podría estar contemplado por la hipótesis del incremento del porcentaje de las precipitaciones de origen mediterráneo en las Demarcaciones Hidrográficas del Segura y Júcar (Miro et al., 2009).

A pesar del aumento del número de días de precipitación $\geq 1 \mathrm{~mm}$, existe un descenso del número de días en los intervalos de $\geq 10 \mathrm{~mm}, \geq 20 \mathrm{~mm}$ y $\geq 50 \mathrm{~mm}$. Este descenso en el número de días con precipitación notable, junto al aumento de las temperaturas y de la evapotranspiración, podría conllevar un descenso drástico de la escorrentía superficial, siendo este proceso más acusado durante el periodo cálido y en aquellos lugares que actualmente concentran la mayor parte de los recursos hídricos, las áreas de montaña (Rasilla et al., 2008). Para la DHS, área donde se encuadra el territorio estudiado, se prevén aumentos significativos de la evapotranspiración de referencia (ET0) para finales del S.XXI, registrándose los principales aumentos en el periodo cálido (desde abril hasta octubre). Los aumentos más significativos se estiman para las zonas más elevadas de la DHS (Ruiz Álvarez et al., 2016). Estos cambios observados y estimados pueden provocar numerosos impactos negativos sobre la oferta de recursos hídricos. De este modo, se hace necesario acometer medidas de planificación y ordenación territorial para paliar las pérdidas económicas que llevarían asociadas, más si cabe en territorios como la Región de Murcia, donde la agricultura y el turismo son dos pilares básicos de su economía.

Por otra parte, con respecto a las tendencias estacionales del número de días de precipitación, es interesante destacar que el verano es la única estación en la cual se producen tendencias negativas en todos los intervalos destacados (figuras 7-10). Además, en la mayor parte de las series analizadas estas tendencias negativas presentan una significancia estadística. La causa de este descenso podría estar en la tendencia general a la reducción de situaciones convectivas, sobre todo en las estaciones de primavera y verano, tal y como ocurre y se ha comprobado en regiones vecinas como la Comunidad Valenciana (Estrella et al., 2006). Por último, en el verano es importante resaltar que en el intervalo de precipitación muy abundante $(\geq 50 \mathrm{~mm})$ se han obtenido algunas tendencias positivas significativas en determinadas series del interior. Por tanto, sería interesante analizar en futuros estudios si se puede estar produciendo una intensificación de los eventos de tormentas severas en el interior del sureste peninsular.

En el resto de estaciones, las tendencias del número de días de lluvia ( $\geq 1 \mathrm{~mm}$ ) son positivas, siendo incluso este ascenso estadísticamente significativo durante la estacional otoñal en numerosas series del interior. Durante el invierno y primavera (figuras 15-22), las tendencias son negativas en el resto de intervalos analizados, siendo estas significativas en la mayor parte de las estaciones en los intervalos de precipitación abundante y muy abundante. En cambio, en la estación otoñal (figuras 11-14) se han obtenido en algunas estaciones del interior tendencias positivas en los intervalos de precipitación $\geq 10 \mathrm{~mm}$ y precipitación abundante. Este hecho podría estar asociado a un aumento de los eventos de lluvias torrenciales durante esta estación, asociadas a Depresiones Aisladas en Niveles Altos (DANA). Un ejemplo claro de estos eventos de lluvias torrenciales en los últimos años lo tenemos en la riada de San Wenceslao (28 de septiembre de 2012), que provocó 
numerosas inundaciones en el sureste de la península ibérica. Además, como se verá en el caso expuesto de la serie "Moratalla Campo de San Juan", se está produciendo un traslado de la estación más húmeda, desde la primavera al otoño.

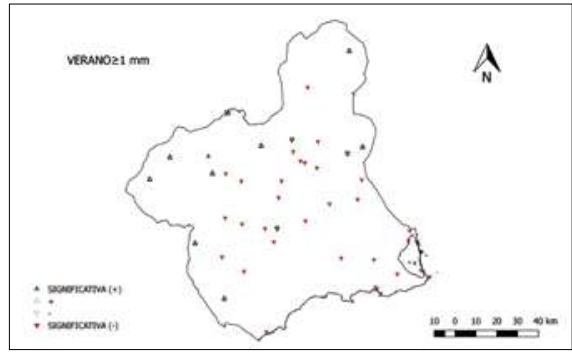

Fig. 8. Tendencia en el verano en el número de días de precipitación $(\geq 10 \mathrm{~mm}$ ) durante el periodo $1947-2016$

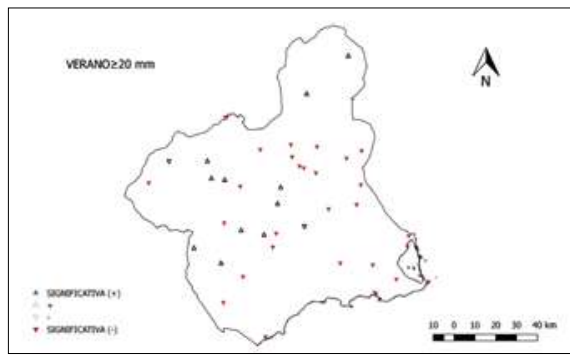

Fig. 10. Tendencia en el verano del número de días de precipitación muy abundante $(\geq 50 \mathrm{~mm})$ durante el periodo 1947-2016

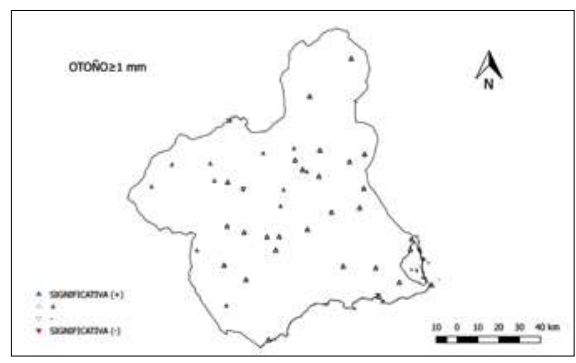

Fig. 7. Tendencia en el verano en el número de días de lluvia ( $\geq 1 \mathrm{~mm}$ ) durante el periodo 1947 2016

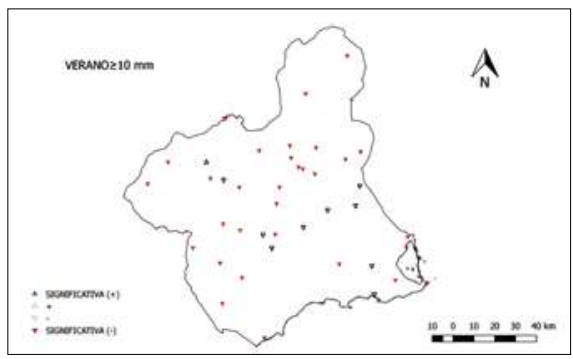

Fig. 9. Tendencia en el verano del número de días de precipitación abundante $(\geq 20 \mathrm{~mm})$ durante el periodo 1947-2016

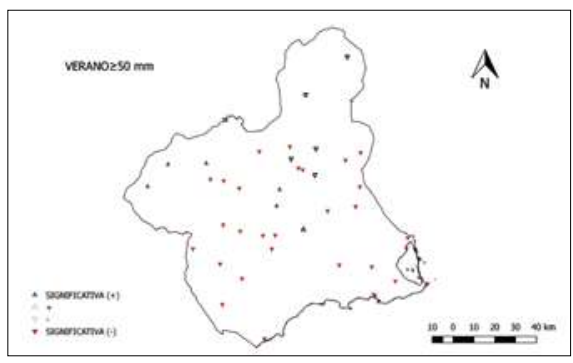

Fig. 11. Tendencia otoñal en el número de días de lluvia ( $\geq 1 \mathrm{~mm}$ ) durante el periodo 1947-2016 
VARIABILIDAD Y CAMBIO CLIMÁTICO

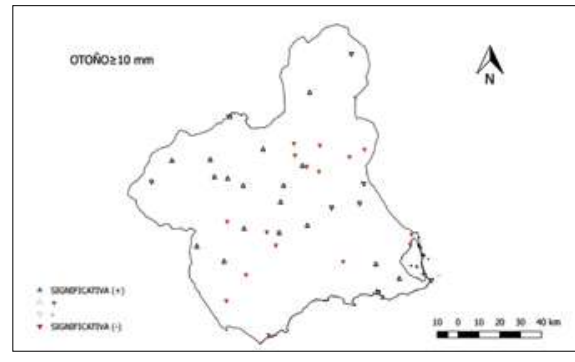

Fig. 13. Tendencia otoñal del número de días de precipitación abundante $(\geq 20 \mathrm{~mm})$ durante el periodo 1947-2016

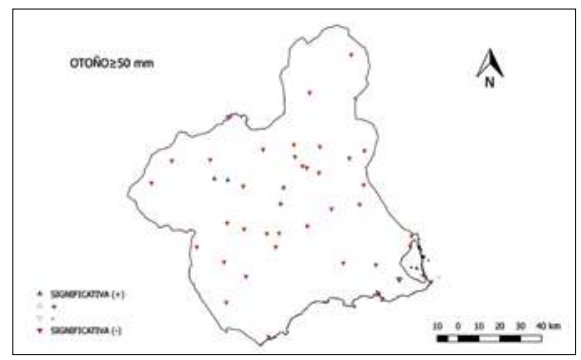

Fig. 15. Tendencia invernal en el número de días de lluvia ( $\geq 1 \mathrm{~mm}$ ) durante el periodo 1947-2016

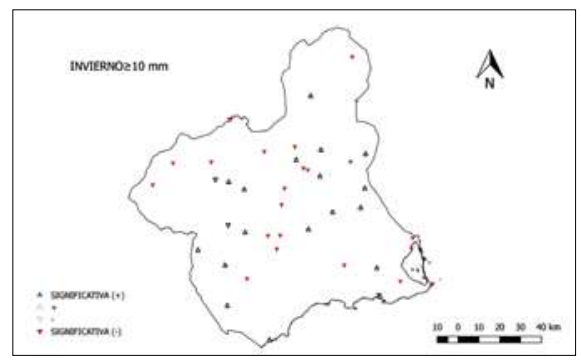

Fig. 17. Tendencia invernal del número de días de precipitación abundante $(\geq 20 \mathrm{~mm})$ durante el periodo 1947-2016
Fig. 12. Tendencia otoñal en el número de días de precipitación $(\geq 10 \mathrm{~mm})$ durante el periodo 1947-2016

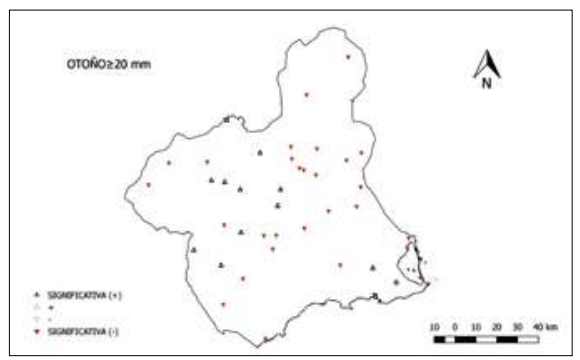

Fig. 14. Tendencia otoñal del número de días de precipitación muy abundante $(\geq 50 \mathrm{~mm})$ durante el periodo 1947-2016

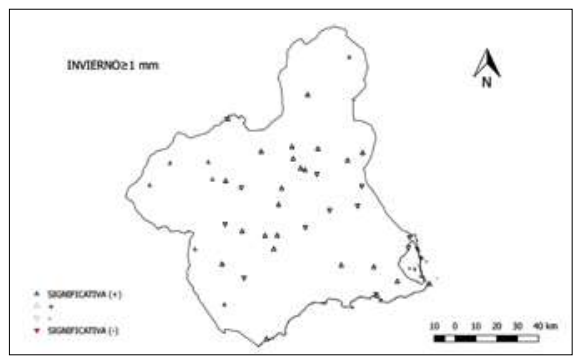

Fig. 16. Tendencia invernal en el número de días de precipitación $(\geq 10 \mathrm{~mm})$ durante el periodo 1947-2016

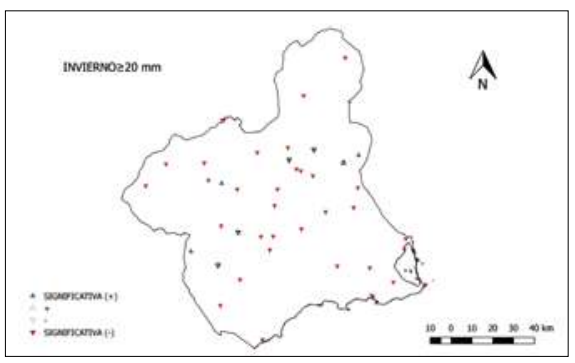

427 


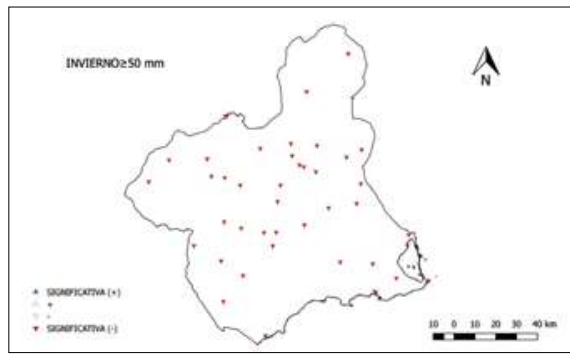

Fig. 18. Tendencia invernal del número de días de precipitación muy abundante $(\geq 50 \mathrm{~mm})$ durante el periodo 1947-2016

Fig. 19. Tendencia primaveral en el número de días de lluvia ( $\geq 1 \mathrm{~mm}$ ) durante el periodo 1947-2016

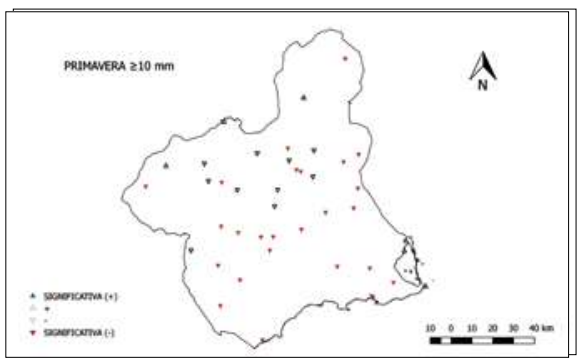

Fig. 21. Tendencia primaveral del número de días de precipitación abundante $(\geq 20 \mathrm{~mm})$ durante el periodo 1947-2016
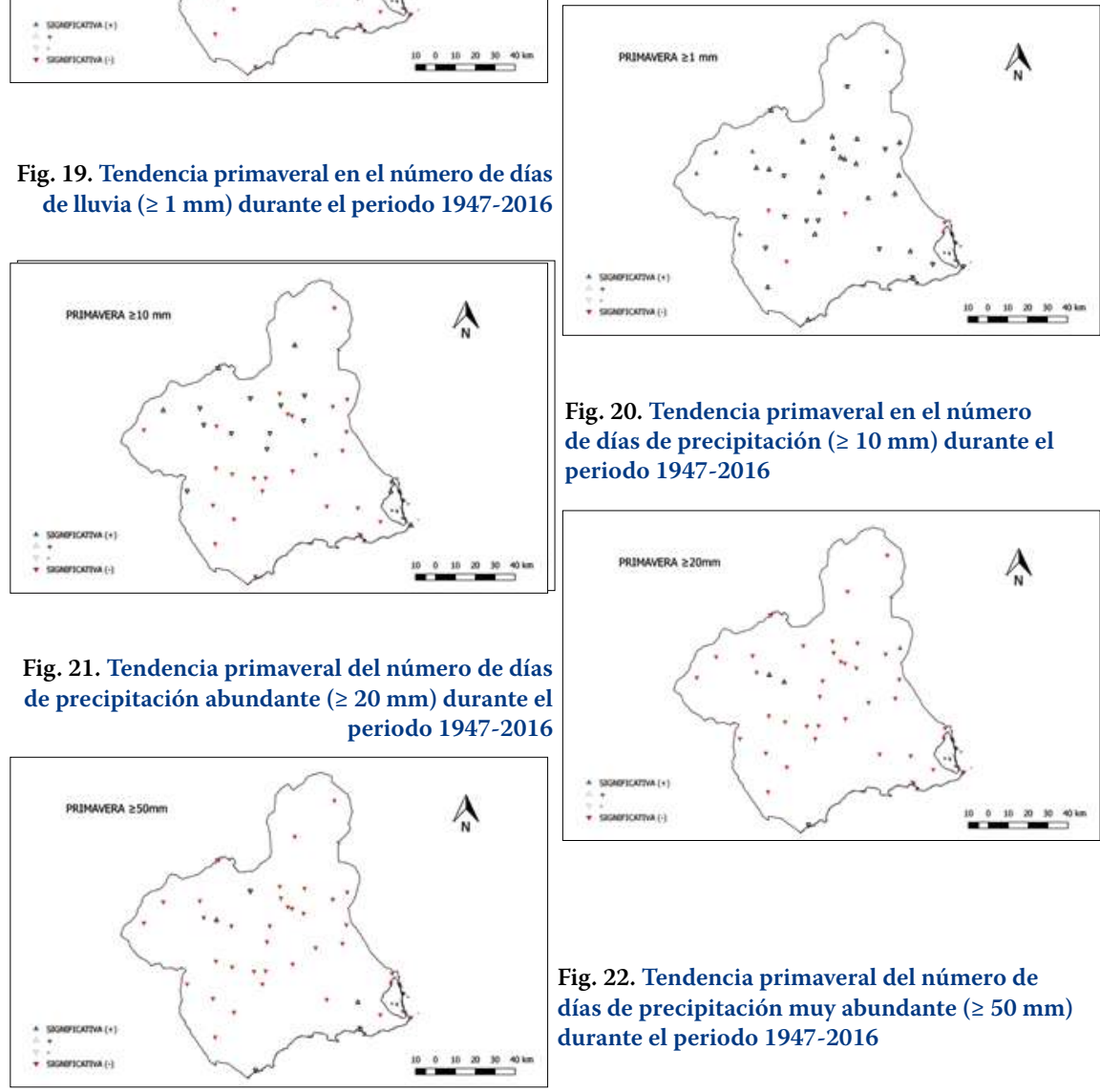

Fig. 20. Tendencia primaveral en el número de días de precipitación $(\geq 10 \mathrm{~mm}$ ) durante el periodo 1947-2016

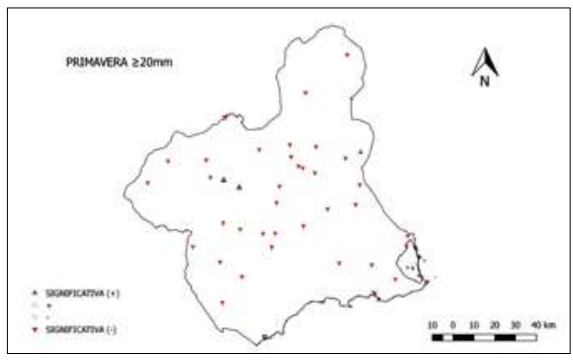

Fig. 22. Tendencia primaveral del número de días de precipitación muy abundante $(\geq 50 \mathrm{~mm})$ durante el periodo 1947-2016

A continuación, se muestran de forma detallada, y como ejemplo, los resultados obtenidos en el análisis de los cambios en los días de precitación de la serie de "Moratalla Campo de San Juan", situada como ya se dijo a 1150 metros de altitud. En la figura 23 se puede apreciar la evolución de las precitaciones durante los equinoccios, mediante la aplicación de una media móvil de 30 años. Tal y como se observa, en los primeros años la primavera era la estación más húmeda, pero en los últimos años esta tendencia está 
invirtiéndose, de modo que el otoño está pasando a ser la estación con mayor porcentaje de precipitación. Este hecho también se denota en el aumento del número de días de precipitación durante el otoño en los intervalos de $\geq 1 \mathrm{~mm}$ y $\geq 10 \mathrm{~mm}$. Este cambio del máximo pluviométrico puede provocar falsas expectativas en los agricultores que se dedican a los cultivos de secano, ya que la tendencia positiva de las precipitaciones durante el otoño puede hacer a los agricultores aumentar la superficie cultivada. Sin embargo, si durante la primavera tienden a descender el número de días de precipitación, los cultivos de secano generarían escasos rendimientos, provocando numerosas perdidas económicas.

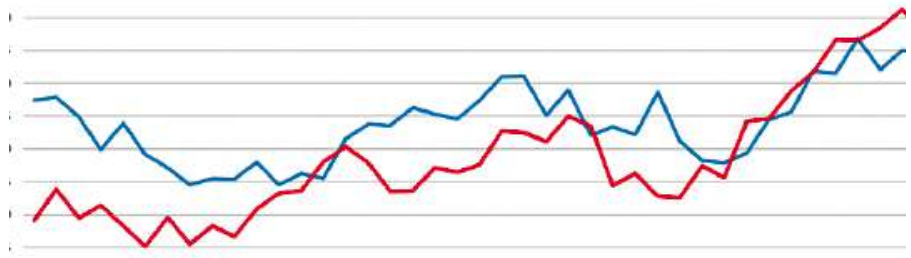

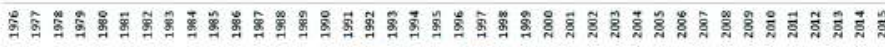

Fig. 23. Evolución de la precipitación (media móvil de 30 años) durante el otoño y la primavera en la serie de "Moratalla Campo de San Juan" durante el periodo 1947 2016.

Por último, se expone la tendencia durante el invierno. En la figura 24 se observa como en el intervalo $\geq 1 \mathrm{~mm}$ la tendencia es positiva. Sin embargo, en el resto de intervalos analizados la tendencia es negativa. Este hecho está provocado por la reducción de las precipitaciones de origen atlántico durante el invierno, ya que esta zona del interior de la Región de Murcia está ligada, sobre todo, a este tipo de precipitaciones. La tendencia negativa de las precipitaciones de origen atlántico también podría influir en la reducción de las nevadas durante el invierno.

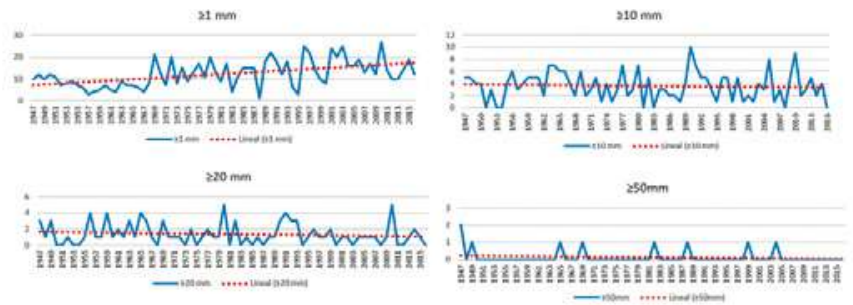

Fig. 24. Cambios y tendencias del número de días de precipitación durante el invierno en la serie "Moratalla Campo de San Juan" durante el periodo 1947-2016

\section{CONCLUSIÓN}

En este trabajo han sido analizados los cambios y las tendencias del número de días de precipitación, en diferentes intervalos $(\geq 1, \geq 10, \geq 20, \geq 50 \mathrm{~mm})$ para una escala tanto anual como estacional. Los resultados obtenidos muestran una tendencia positiva para el intervalo $\geq 1 \mathrm{~mm}$, siendo significativa en algunos observatorios del interior. Sin 
embargo, en el resto de intervalos analizados la tendencia es negativa, siendo significativa en los intervalos de precitación abundante y muy abundante. Una de las causas de estos cambios podría ser la disminución de las lluvias de origen atlántico y el aumento del porcentaje de las precipitaciones de origen mediterráneo. Estos cambios podrían conllevar importantes connotaciones negativas en el territorio analizado, provocando graves impactos en la oferta natural de recursos hídricos. Por lo tanto, se deberían articular medidas de planificación y ordenación territorial, con el fin de adaptarse a estos cambios, y minimizar al máximo las pérdidas económicas asociadas.

A escala estacional, se han obtenido tendencias negativas significativas durante la estación estival en todos los intervalos analizados, que podrían estar asociados a un descenso de los fenómenos convectivos. El resto de estaciones sí presentan un aumento significativo en el número de días de lluvia $\geq 1 \mathrm{~mm}$. Este aumento es estadísticamente significativo en algunas series del interior. En el resto de intervalos las tendencias son negativas en todas las estaciones, al igual que ocurre a escala anual, exceptuando el intervalo $\geq 10 \mathrm{~mm}$ en otoño, en el cual algunas series del interior presentan tendencias positivas. Por otra parte, cabe reseñar que se está produciendo un cambio del máximo pluviométrico estacional desde la primavera al otoño. Este factor puede provocar un sentimiento de falsa seguridad en los agricultores dedicados a los cultivos de secano. Por último, el descenso del número de días de precipitación abundante y muy abundante, en el interior regional, puede tener impactos graves sobre la oferta de recursos hídricos, y también en la recarga de los acuíferos. De este modo, en los próximos años se pueden agudizar los conflictos territoriales y sociopolíticos si no se lleva a cabo una gestión eficaz de los recursos hídricos, ya que en estas zonas de interior se concentran la mayor parte de las reservas hídricas de este territorio.

Para finalizar, como líneas de trabajo futuras, se pretende estudiar la correlación de los cambios en el número de días de precipitación con diferentes índices de teleconexión (WeMO, NAO y MO). Otro objetivo es analizar los cambios en los diferentes intervalos de precipitación a escala mensual, así como examinar la percepción social que tiene la población sobre estos cambios.

\section{REFERENCIAS}

- Brunet, M., Sigró, J., Jones, P.D., Saladié, O., Aguilar, E., Moberg, A., Lister, D., Walther, A.,2007: Long-term changes in extreme temperatures and precipitation in Spain. Institut d'Estudis Catalans, 3, 331-242.

- De Luis, M.; Raventós, J.; González-Hidalgo, J.C.; Sánchez, J.R. y Cortina, J., 2000: Spatial analysis of rainfall trends in the region of Valencia (East Spain). International Journal of Climatology, 20, 1451-1469.

- Esteban-Parra, M.J, Rodrigo, F.S. y Castro-Díez Y., 1998: Spatial and temporal patterns of precipitacion in Spain for the period 1880- 1992. International Journal of Climatology, 18, 15571574.

- Estrella, M.J., Miro, J.J., y Millán, M., 2006: Análisis de tendencia de la precipitación por situaciones convectivas en la Comunidad Valenciana (1959-2004). En: Cuadrat Prats et al. (Eds.): Clima, Sociedad y Medio Ambiente. Publicaciones de la Asociación Española de Climatología (AEC) Serie A, nº5. Zaragoza, 1-12. 
Gallego, M.C. et al.,2011: Trends in frequency indices of daily precipitation over the Iberian Peninsula during the last century. Journal of Geophysical Research, 116.

- García Marín, R. y Conesa García, C. 2006: Secuencias pluviométricas secas de larga duración en la cuenca del Guadalentín (Murcia- Almería). En: Cuadrat Prats et al. (Eds.): Clima, Sociedad y Medio Ambiente. Publicaciones de la Asociación Española de Climatología (AEC) Serie A, n5. Zaragoza, 371-382.

- García Marín, R. y Calvo García-Tornel, F. 2008: Frecuencia y evolución de rachas secas en la Cuenca del Guadalentín (Sureste de España). Boletín de la Asociación de Geógrafos Españoles, 48, 71-89.

- González-Hidalgo, J.C.; De Luis, M.; Raventos, J. y Sánchez, R. ,2003: Daily rainfall trend in the Valencia Region of Spain. Theoretical and Applied Climatology 75,117-130.

- Hoerling, M., Eischeid, J., Perlwitz, J., Quan, X.W., Zhang, T., Pegion, P. ,2012: On the increased frequency of Mediterranean drought. Journal of Climate, 25, 2146 - 2161.

- Kendall, S. (1976): Time Series, 2nd ed. Oxford Univ. Press, New York, 198 pp.

- Martín-Vide, J. y Olcina, J. ,2001: Climas y tiempos de España. Alianza Editorial. Madrid, 258 pp.

- Mestre, O., Domonkos, P., Picard, F., Auer, I., Robin, S., Lebarbier, E., Klancar, M.,2013: HOMER: a homogenization software-methods and applications.

- Miró Pérez, J., Estrella Navarro, M.J., Pastor, F. y Millán, M., 2009: Análisis comparativo de tendencias en la precipitación, por distintos inputs, entre los dominios hidrológicos del Segura y del Júcar (1958-2008). Investigaciones Geográficas, 49, 129-157. Instituto Universitario de Geografía, Universidad de Alicante.

- Rasilla, D. Garmendia, C., García, J.C., 2008: Proyecciones de cambio climático sobre las aguas superficiales en la península ibérica. En: Sigró Rodríguez, J.; Brunet India, M.; Aguilar Anfrons, E. (eds.). Cambio climático regional y sus impactos. Tarragona: Asociación Española de Climatología, 587-597

- Ríos Cornejo, D., Penas, A. y Del Río, S. ,2013: Comparative analysis of precipitation trends in continental spain over the period 1961-2010. International Journal of Geobotanical Research, 3, 1-18.

- Ruiz Álvarez, M., Gomariz Castillo, F. y Alonso Sarría, F., 2016: Impacto del cambio climático sobre la evapotranspiración de referencia. XV Coloquio Ibérico de Geografía: Retos y tendencias de la geografía ibérica, Murcia, 357-366.

- Ruiz Álvarez, V., 2017: Analysis of precipitations trends in the Region of Murcia (Southeast Spain) over the period 1956-2015. 6th International Conference on Meteorology and Climatology of the Mediterranean, February 20-22, Zagreb, Croacia.

- Serrano, A., Mateos, V.L. y García, J.A.,1999: Trend analysis of monthly precipitation over the iberian peninsula for the period 1921-1995. Physic and Chemistry of the Earth Part B, 24 (1-2), 85-90.

- Sousa, P.M., Trigo, R.M., Aizpurua P., Nieto R, Gimeno L. y Garcia-Herrera, R.,2011: Trends and extremes of drought indices throughout the 20th century in the Mediterranean. Natural Hazards Earth System Sciences, 11, 11-33. 